\title{
Manipulation of Cell-Cell Adhesion Using Bowtie-Shaped Microwells
}

\author{
Celeste M. Nelson, Wendy F. Liu, and Christopher S. Chen
}

\begin{abstract}
Summary
Traditional methods to study cell-cell adhesion have been limited by their inability to manipulate cell-cell interactions without simultaneously affecting other microenvironmental factors. Here we describe a novel method that enables the culture of cells with precise simultaneous control of both cell-cell and cell-substratum adhesion. Using microfabricated stamps of poly(dimethylsiloxane), we construct bowtie-shaped agarose microwells into which cells can be cultured. The degree to which cells spread is controlled by the size of the microwell; cell-cell contacts form between neighboring cells within the microwell. This chapter describes the details of stamp fabrication, agarose microwell construction, and cell culture in micropatterned substrata.
\end{abstract}

Key Words: Cell-cell interaction; cadherin; microfabrication.

\section{Introduction}

Micropatterning has emerged as a valuable family of techniques to precisely control the cellular adhesive environment (1). Adherent cells monitor and respond to their surroundings using receptors on their surfaces that bind to ligands both in the extracellular matrix (ECM) and on neighboring cells. Micropatterning allows the investigator to present these cues in spatially organized arrangements to cells in culture with micrometer precision, providing a means to explore structure-function relationships between cells and their extracellular space. Biological studies using micropatterning have examined the roles of cell-ECM $(2,3)$ and cell-cell (4-6) interactions in the control of cellular function with a specificity that could not have been accomplished using traditional techniques.

Cells adhere to the surrounding ECM and neighboring cells through several classes of transmembrane proteins that mechanically link to the intracellular

From: Methods in Molecular Biology, vol. 370:

Adhesion Protein Protocols, Second Edition

Edited by: A. S. Coutts $(\subset$ Humana Press Inc., Totowa, NJ 
cytoskeleton. Cell-ECM and cell-cell binding interactions play an important role in regulating a number of processes, including proliferation, differentiation, and gene expression. Cell adhesion to the ECM involves binding and clustering of integrins and cell spreading against the matrix. Both integrin binding and changes in cell shape appear to be important mediators of cell signaling and function (7). While many techniques have been developed to manipulate cell-ECM interactions $(8,9)$, controlling cell-cell interactions is less straightforward. Early studies examining the effects of cell-cell interactions on cellular function were limited to observations of cells grown in typical culture conditions. Cells cultured at a low density in Petri dishes form few cell-cell adhesions, whereas cells that grow to a high density form many more cell-cell adhesions; observed differences between the behaviors of cells at low and high density were then correlated to the effects of cell-cell adhesion $(10,11)$. In other studies, the aggregation of cells in suspension was used to determine relative cell-cell interaction strength (12). One confounding problem with the interpretation of these experiments is that the density or aggregation context also modulates other environmental cues. For example, the concentration of secreted soluble factors in the media may be greater for high-density cultures. Adhesive factors or cell spreading against the surface also varies: typically, cells at a low density are spread to a greater extent than cells at a higher density. Because cadherins, the primary adhesive molecules involved in cell-cell adhesion, are dependent on extracellular calcium, engagement of cadherin contacts can be induced by removal and then addition of extracellular calcium into the media-also known as a "calcium switch" assay (13). Again, however, decreasing calcium concentration causes cells to round up and alters cell-ECM adhesion. As many investigations have shown, cell-ECM adhesion alone directly affects cell function, perhaps through biochemical, cytoskeletal, or mechanical changes $(2,8)$. An alternative method used to study cell-cell contacts applies isolated plasma membranes to cells in culture, which was observed to mimic the effects of high cell density (14). After the identification of specific families of cell-cell adhesion receptors (15), some groups have more recently begun to examine the effects of cadherin engagement using protein-coated beads or surfaces $(\mathbf{1 6}, \mathbf{1 7})$. Whereas studies have shown that contact to a cadherin-coated bead can induce biological responses similar to cadherin-mediated contact to neighboring cells (18), the physiological relevance and biological significance of engaging a cadherin molecule attached to a bead or surface are uncertain.

Here we describe a novel microfabrication method used to isolate the effects of cell-cell contact while holding constant cell-ECM adhesion by controlling the degree to which cells spread against the underlying substratum. Cells are seeded into bowtie-shaped microwells so that at most one pair of cells is cultured within each microwell, and thus each cell only contacts a single neighboring cell. 
The degree of cell spreading is determined by the area of the microwell. This technique allows the control of cell-cell contacts without changing the effective culture density, the aggregation context, or the extracellular ion concentration. Furthermore, the cadherin contacts are formed with neighboring cells and are therefore physiologically relevant. Studies using these microwells have demonstrated that some effects traditionally attributed to cell-cell contact are actually caused by contact-mediated changes in cell-ECM adhesion and that the regularity of cell-cell adhesions formed in this assay can amplify the specific effects of cell-cell contact that might otherwise be lost in the heterogeneity of typical culture conditions $(5,6)$. Here we describe the procedure used to produce bowtie-shaped microwells. We describe the photolithographic methods used to generate the master templates from which rubber stamps are cast. The stamps are then used to produce agarose substrata on glass, onto which cells can be cultured and studied.

\section{Materials}

1. Silicon wafers (2- to 3-in. diameter, test grade, 13-17 mils, $<100>$ orientation, $1-10$ $\Omega \mathrm{cm}$; Silicon Sense, Nashua, NH).

2. SU-8-negative photoresist (MicroChem Corp., Newton, MA).

3. Spin-coater (Headway Research, Inc., Garland, TX).

4. Mask aligner (Karl Suss, Waterbury Center, VT).

5. Propyleneglycol methyl ether acetate developer (Sigma-Aldrich, St. Louis, MO).

6. Digital hotplate (Barnstead Thermolyne, Dubuque, IA).

7. Vacuum dessicator (Fisher Scientific, Hampton, NH).

8. (Tridecafluoro-1,1,2,2,-tetrahydrooctyl)-1-trichlorosilane (United Chemical Technologies, Bristol, PA).

9. Glass slides (Fisher Scientific).

10. Poly(dimethylsiloxane) (PDMS; Sylgard 184; Ellsworth Adhesives, Germantown, $\mathrm{WI})$.

11. UVO cleaner (Jelight Corporation, Irvine, CA).

12. Superfrost glass slides (Fisher Scientific).

13. Agarose (ISCBioExpress, Kaysville, UT).

14. Ethanol (200 proof).

15. Fibronectin (Invitrogen, Carlsbad, CA).

16. Phosphate-buffered saline (PBS).

\section{Methods}

Our goal was to develop a technique to control cell-cell adhesion while simultaneously controlling cell spreading. Our design is a substratum consisting of bowtie-shaped islands of matrix surrounded by nonadhesive regions. Each cell of a pair adheres and spreads to fill one half of the bowtie-shaped region; the cells contact each other at the central constriction of the bowtie. We initially screened 


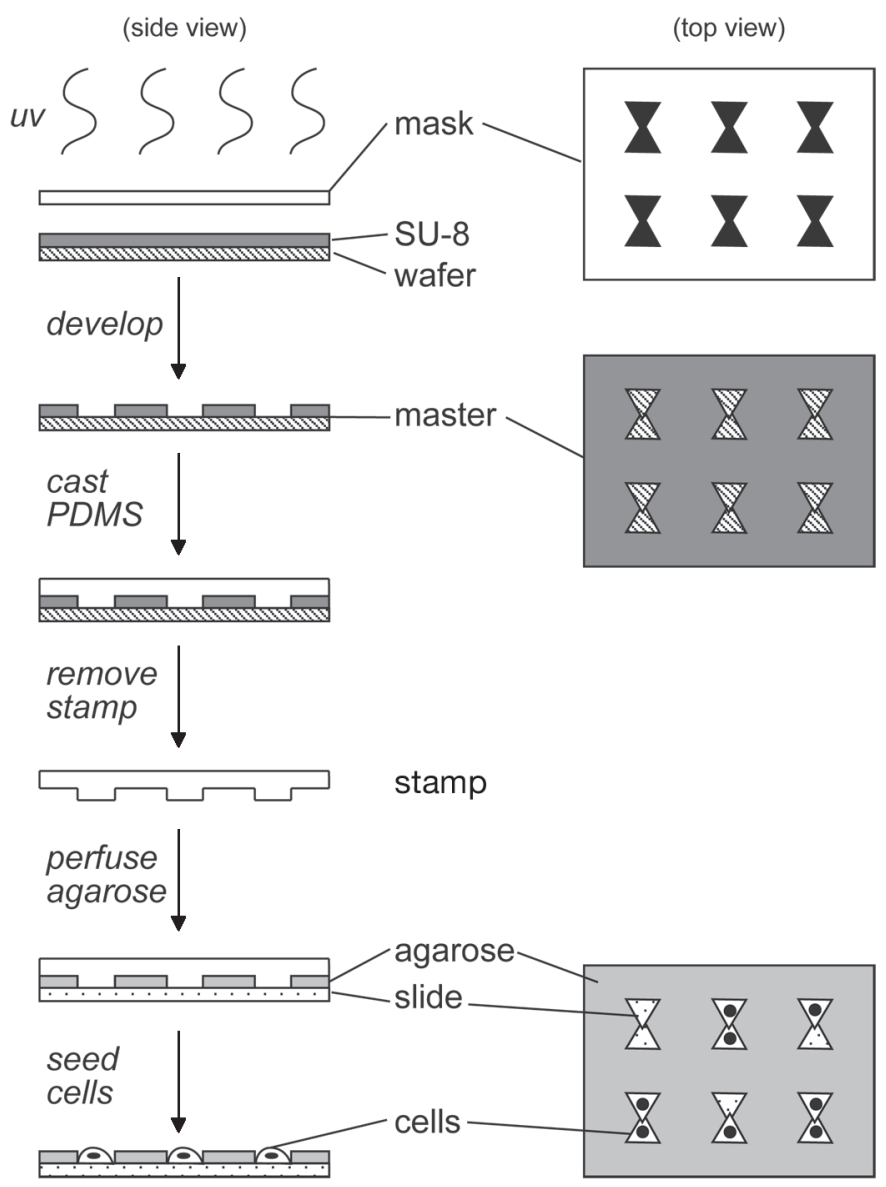

Fig. 1. Schematic of patterning process. PDMS, poly(dimethyl siloxane).

several shapes, including overlapping circles and squares, and found that endothelial cells spread best to fill the triangular features comprising one half of the bowtie-shaped patterns. We have not tested all geometries, and other cell types may spread preferentially in other shapes. We found that in order to precisely control the area of cell-cell contact and prevent the contact from expanding, we had to culture cells within shallow wells of nonadhesive material; molding a thin film of agarose gel worked well for our purposes. The methods described here outline the following (Fig. 1):

1. Fabrication of the master.

2. Molding and treatment of the stamp.

3. Construction of the agarose wells.

4. Coating of the wells and plating of cells. 


\subsection{Master Fabrication}

1. Spin SU-8 photoresist on the shiny side of a clean silicon wafer for the specified rate and duration, depending on the desired height of microwells (a table with spin parameters for various desired thicknesses of resist can be found on the MicroChem website: http://www.microchem.com/products/su_eight.htm). There are many kinds of photoresist, but they all fall into two classes: positive and negative. For positive resists, the region to be removed is exposed to UV light, which alters the chemical structure of the resist and thereby renders it soluble to a particular developing solvent. The exposed resist is washed away during the developing step (see step 5), leaving a pattern of raised features on the wafer identical to the dark regions on the photomask. For negative resists, the region to be removed is blocked from exposure to UV light, which polymerizes the resist and thereby renders it insoluble to the developing solvent. The exposed resist remains on the wafer during the developing step, with the unexposed regions washing away, leaving a pattern of raised features identical to the clear regions on the photomask. Therefore, the choice of resist type (positive or negative) and design of photomask (darkfield or clear-field) go hand-in-hand. SU-8 is a negative photoresist, therefore the dark regions (bowtie shapes) on the photomask used will correspond to the eventual agarose microwells. The height of the microwells is determined by the thickness of the resist spun onto the wafer; negative resists in general, and SU-8 in particular, are suitable for making thick layers. We have found that resist thicknesses of $10-50 \mu \mathrm{m}$ are needed to construct the agarose microwells.

2. Place the wafer on a digital hot plate for "soft bake" to evaporate solvent and dry the resist onto the surface of the wafer. The duration and temperature of baking depend on the thickness of the SU-8; a table of these parameters can also be found on the MicroChem website.

3. Place the wafer and chrome mask in the mask aligner, chrome-side down, and expose with approx $10-12 \mathrm{~mJ} / \mathrm{cm}^{2}$ of UV irradiation per $\mu \mathrm{m}$ thickness of resist (e.g., if making $50-\mu \mathrm{m}$-thick master, use $600 \mathrm{~mJ} / \mathrm{cm}^{2}$ ).

4. Remove the mask and place the wafer on a digital hot plate set at $90^{\circ} \mathrm{C}$ for at least 5 min ("hard bake").

5. Immerse the master into a bath of propyleneglycol methyl ether acetate developing solution and shake gently until the pattern can be observed (approx $2 \mathrm{~min}$, but time will depend on thickness of the resist).

6. Dry the master under a steady stream of compressed nitrogen gas.

7. To aid in the later removal of PDMS, place the silicon master in a vacuum dessicator with a glass slide containing a drop of (tridecafluoro-1,1,2,2,-tetrahydrooctyl)-1-trichlorosilane. Evacuate the chamber. Sufficient silanization can be achieved either by exposing to vacuum overnight or by isolating the chamber for $2 \mathrm{~h}$ after evacuation.

\subsection{Molding and Treatment of the Stamp}

1. Prepare $30 \mathrm{~g}$ 10:1 (w/w) PDMS polymer:curing agent solution. Mix thoroughly and remove air bubbles by placing the mixture in a vacuum dessicator. This volume of PDMS is sufficient for a 2- or 3-in. silicon master in a standard 100-mm Petri dish. 
2. Pour degassed PDMS mixture onto silicon master.

3. Bake at $60^{\circ} \mathrm{C}$ for $2 \mathrm{~h}$.

4. Carefully peel hardened PDMS stamp from silicon master. Silicon masters can be reused indefinitely.

5. Treat stamp for $5 \mathrm{~min}$ in UV/ozone cleaner. Stamps should be used to make agarose wells on the same day as UV/ozone treatment (see Note 1).

\subsection{Construction of Agarose Wells}

The goal of this procedure is to fill the channels between the PDMS stamp and the glass slide with agarose. When the stamp is removed, the agarose "walls" surround glass-bottomed "wells," into which the cells will later be seeded. There are two major obstacles to filling PDMS channels with agarose: (1) the PDMS channels are quite hydrophobic and prevent the flow of aqueous solutions through them, and (2) aqueous agarose solutions are quite viscous, have a high surface tension, and do not flow very readily. The first obstacle is addressed (Subheading 3.2., step 5) by treating the PDMS stamp in the UV/ozone cleaner, which modifies the surface of the stamp to render it more hydrophilic and helps the solution flow through the channels. The second obstacle is addressed by adding ethanol to the agarose solution to decrease both its viscosity and surface tension. The procedure described below was arrived at empirically in our laboratory, and certain details might need to be altered depending on experimental conditions.

1. Prepare a solution of $1 \%$ agarose in distilled water. Heat to $80^{\circ} \mathrm{C}$. The stock solution of $1 \%$ agarose can be reused, but should be stored in a tightly sealed container to prevent evaporation or contamination.

2. Mix six parts $1 \%$ agarose solution with four parts 200-proof ethanol. Because ethanol evaporates readily, the agarose/ethanol solution should be prepared fresh for each batch of microwells (see Note 2).

3. Seal UV/ozone-treated stamp against Superfrost slide, feature-side down.

4. Pipet a drop of the hot agarose/ethanol solution against the side of the sealed stamp. Allow the solution to perfuse through the channels formed between the stamp and the slide. The volume of agarose/ethanol solution required per sample will depend on the size of the stamp. Adequate perfusion can be obtained with stamps approx $0.5 \mathrm{~cm}$ wide.

5. Dry the slides by placing in a vacuum dessicator for approx $10 \mathrm{~min}$, or until dry. During this time the agarose will gel and the solvent will evaporate, leaving a thin film of agarose in the desired pattern on the glass slide (see Note 3).

6. Carefully remove the stamp from the slide with fine-tipped forceps. During removal, avoid any lateral movement of the stamp, which may smear the agarose film and deform or destroy the microwells (in other words, pull the stamp straight up). Each microwell will consist of a base of bare glass surrounded by walls of agarose and can be easily inspected for defects using a standard microscope (Fig. 2A). 

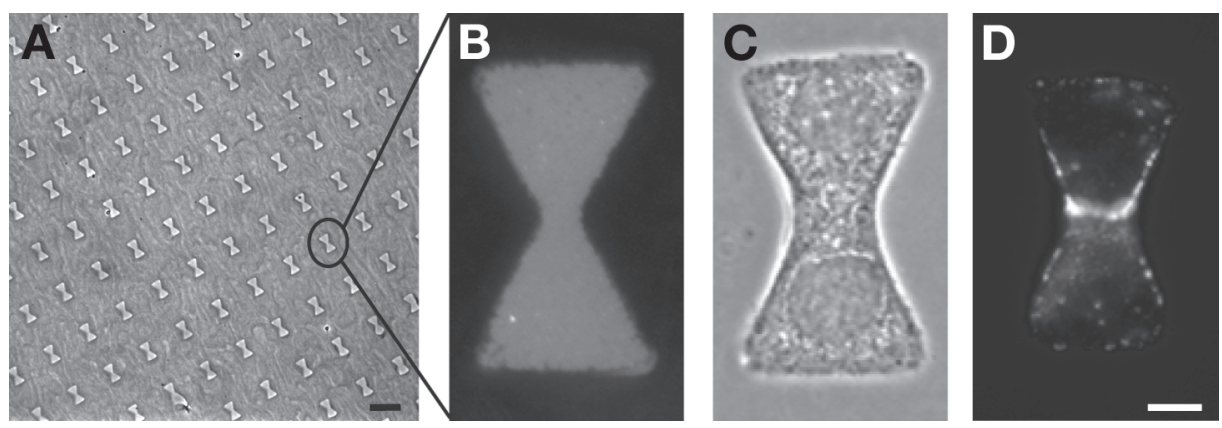

Fig. 2. Bowtie-shaped agarose microwells restrict protein adsorption, cell attachment and spreading, and cell-cell adhesion. Shown are (A) phase contrast image of agarose wells, (B) fluorescence image of staining for fibronectin, (C) phase contrast image of cells, and (D) $\beta$-catenin in bowtie-shaped microwells. A: Bar $=50 \mu \mathrm{m}$; B-D: Bar $=10 \mu \mathrm{m}$.

\subsection{Coating and Cell Culture}

After construction of the agarose microwells, cells can be seeded directly into the untreated glass wells or the wells may first be coated with a specific ECM protein to which the cells can subsequently adhere. For endothelial cells, we typically coat the wells with a solution of fibronectin. Because agarose is a hydrogel, it is relatively resistant to protein adsorption, so fibronectin adsorbs only to the bare glass bases of the microwells (5) (Fig. 2B). Because agarose resists adsorption of ECM proteins necessary for cell adhesion, it consequently resists cell attachment; when cells are seeded onto the substratum, they attach exclusively to the glass-bottomed wells (see Note 4).

1. Place agarose-coated slides in $70 \%$ ethanol solution to sterilize for cell culture.

2. Rinse twice with PBS.

3. Coat with $25 \mu \mathrm{g} / \mathrm{mL}$ solution of fibronectin in PBS for $1-2 \mathrm{~h}$ at room temperature.

4. Rinse twice with PBS.

5. Plate cells on agarose-coated slides in normal culture media. Allow cells to adhere in wells. Rinse to remove excess cells (Fig. 2C,D).

\subsection{Experimental Analysis}

We have found that a typical substratum will yield several hundred bowtieshaped cultures, some of which contain a pair of contacting cells, and some of which contain a single cell without a neighbor. Each substratum sample therefore consists of a subpopulation of cells (the single cells) that acts as an internal control. However, because of the small number of cells and the heterogeneity of the bowties on a single substratum, experimental analysis is limited primarily to in situ techniques. We have used the bowtie microwells to examine the effects of cell-cell contact on proliferation by staining for BrdU incorporation $(5,19)$ and on cell-ECM adhesion by immunofluorescence analysis (20). One should 
also be able to combine the bowtie microwells with in situ hybridization or in situ reverse transcription-polymerase chain reaction (RT/PCR) to study changes in gene expression. Theoretically, one should be able to scale up the technique; larger patterned areas or a higher percentage of patterned pairs of cells would allow one to obtain enough material for bulk population analysis (via Western blotting, etc.).

The method is adaptable for different types of cells. In addition to a number of types of endothelial cells, we have successfully cultured smooth muscle cells, fibroblasts, and epithelial cells in the bowtie microwells (see Note 5). The agarose bowties are best suited for experiments of short duration (less than a few days), because the agarose layer tends to detach from the glass over extended periods in aqueous solutions, such as culture media (21) (see Note 6).

\section{Notes}

1. Stamps can be reused four to five times.

2. If the final ethanol concentration is too high, agarose will precipitate out of solution and the film is no longer nonadhesive.

3. The agarose layer will detach from the slide if it is not first dried completely.

4. Because of chemical modifications, low-melt agarose is not resistant to protein adsorption. Thus, cells would adhere everywhere to such a substratum.

5. Some of the patterning parameters may need to be altered when working with different types of cells. We have found that longer drying times are needed to increase the stiffness of the agarose gel for some cell lines (such as normal rat kidney epithelial cells).

6. For experiments that require patterning cells over longer time scales, we have developed an alternative method of constructing wells with walls of nonadhesive polyacrylamide gel (21). These substrata will maintain their pattern for at least 2 mo.

\section{Acknowledgments}

This work was supported by grants from the NIH, the Whitaker Foundation, and the Defense Advanced Research Projects Agency. C.M.N. acknowledges financial support from the Whitaker Foundation, and W.F.L. was supported by the National Science Foundation.

\section{References}

1. Folch, A. and Toner, M. (2000) Microengineering of cellular interactions. Annu. Rev. Biomed. Eng. 2, 227-256.

2. Chen, C. S., Mrksich, M., Huang, S., Whitesides, G. M., and Ingber, D. E. (1997) Geometric control of cell life and death. Science 276, 1425-1428.

3. McBeath, R., Pirone, D. M., Nelson, C. M., Bhadriraju, K., and Chen, C. S. (2004) Cell shape, cytoskeletal tension, and RhoA regulate stem cell lineage commitment. Dev. Cell 6, 483-495. 
4. Bhatia, S. N., Balis, U. J., Yarmush, M. L., and Toner, M. (1999) Effect of cellcell interactions in preservation of cellular phenotype: cocultivation of hepatocytes and nonparenchymal cells. FASEB J. 13, 1883-1900.

5. Nelson, C. M. and Chen, C. S. (2002) Cell-cell signaling by direct contact increases cell proliferation via a PI3K-dependent signal. FEBS Lett. 514, 238-242.

6. Nelson, C. M. and Chen, C. S. (2003) VE-cadherin simultaneously stimulates and inhibits cell proliferation by altering cytoskeletal structure and tension. J. Cell Sci. 116, 3571-3581.

7. Huang, S. and Ingber, D. E. (1999) The structural and mechanical complexity of cell-growth control. Nat. Cell Biol. 1, E131-138.

8. Folkman, J. and Moscona, A. (1978) Role of cell shape in growth control. Nature 273, 345-349.

9. Singhvi, R., et al. (1994) Engineering cell shape and function. Science 264, 696698.

10. Holley, R. W. and Kiernan, J. A. (1968) "Contact inhibition" of cell division in 3 T3 cells. Proc. Natl. Acad. Sci. USA 60,300-304.

11. Stoker, M. G. and Rubin, H. (1967) Density dependent inhibition of cell growth in culture. Nature 215, 171-172.

12. Steinberg, M. S. and Takeichi, M. (1994) Experimental specification of cell sorting, tissue spreading, and specific spatial patterning by quantitative differences in cadherin expression. Proc. Natl. Acad. Sci. USA 91, 206-209.

13. Gonzalez-Mariscal, L., Chavez de Ramirez, B., and Cereijido, M. (1985) Tight junction formation in cultured epithelial cells (MDCK). J. Membr. Biol. 86, 113-125.

14. Nakamura, T., Yoshimoto, K., Nakayama, Y., Tomita, Y., and Ichihara, A. (1983) Reciprocal modulation of growth and differentiated functions of mature rat hepatocytes in primary culture by cell—cell contact and cell membranes. Proc. Natl. Acad. Sci. USA 80, 7229-7233.

15. Takeichi, M. (1977) Functional correlation between cell adhesive properties and some cell surface proteins. J. Cell Biol. 75, 464-474.

16. Kovacs, E. M., Ali, R. G., McCormack, A. J., and Yap, A. S. (2002) E-cadherin homophilic ligation directly signals through Rac and phosphatidylinositol 3-kinase to regulate adhesive contacts. J. Biol. Chem. 277, 6708-6718.

17. Levenberg, S., Katz, B. Z., Yamada, K. M., and Geiger, B. (1998) Long-range and selective autoregulation of cell-cell or cell-matrix adhesions by cadherin or integrin ligands. J. Cell Sci. 111 (Pt. 3), 347-357.

18. Lambert, M., Padilla, F., and Mege, R. M. (2000) Immobilized dimers of N-cadherin-Fc chimera mimic cadherin-mediated cell contact formation: contribution of both outside-in and inside-out signals. J. Cell Sci. 113, 2207-2219.

19. Liu, W. F., Nelson, C. M., Pirone, D. M., and Chen, C. S. (2006) E-cadherin engagement stimulates proliferation via Rac1. J. Cell Biol. 173, 431-441.

20. Nelson, C. M., Pirone, D. M., Tan, J. L., and Chen, C. S. (2004) Vascular endothelial-cadherin regulates cytoskeletal tension, cell spreading, and focal adhesions by stimulating RhoA. Mol. Biol. Cell 15, 2943-2953.

21. Nelson, C. M., Raghavan, S., Tan, J. L., and Chen, C. S. (2003) Degradation of micropatterned surfaces by cell-dependent and -independent processes. Langmuir 19, 1493-1499. 
